\title{
ALM models based on second order stochastic dominance
}

\author{
Maram Alwohaibi $^{1}$ (D) Diana Roman ${ }^{2}$
}

Received: 14 November 2016 / Accepted: 7 February 2018 / Published online: 8 March 2018

(C) The Author(s) 2018. This article is an open access publication

\begin{abstract}
We propose asset and liability management models in which the risk of underfunding is modelled based on the concept of stochastic dominance. Investment decisions are taken such that the distribution of the funding ratio, that is, the ratio of asset to liabilities, is non-dominated with respect to second order stochastic dominance. In addition, the funding ratio distribution is close in an optimal sense to a user-specified target distribution. Interesting results are obtained when the target distribution is degenerate; in this case, we can obtain equivalent risk minimisation models, with risk defined as expected shortfall or as worst case loss. As an application, we consider the financial planning problem of a defined benefit pension fund in Saudi Arabia.
\end{abstract}

\section{Introduction and motivation}

To solve a pension fund's asset and liability management (ALM) problem means to determine decisions on one or more among the following: allocation of the assets, contributions rate, level of payments, which are optimal in some sense. Usually, the objective is long term increase in wealth while satisfying solvency requirements at all times. The solvency of a fund is measured by the funding ratio, that is, the ratio of assets to liabilities (Gallo 2009).

The outcome of such decisions depends on parameters whose future value is not known with certainty at decision time; such parameters include asset returns, future lia-

\footnotetext{
\aram Alwohaibi

Maram.Alwohaibi@brunel.ac.uk

1 Department of Mathematics, Brunel University London, Uxbridge UB8 3PH, UK

2 Brunel University College of Engineering Design and Physical Sciences, London, UK
} 
bilities and contributions. In the stochastic programming (SP) approach, the stochastic processes of interest are discretized and represented through a finite set of scenarios.

While pure asset allocation problems are usually modelled as single period, in the case when liabilities are present, a multi-stage setting is adopted.

The basic concepts of ALM models using Stochastic Programming were developed by Kallberg et al. (1982) and Kusy and Ziemba (1986), please also see Pirbhai et al. (2003) and references within. The first commercial application of an asset-liability model was reported in the literature by Carino et al. (1994) and Carino and Ziemba (1998) for the second largest Japanese insurance company. Other examples include Consigli and Dempster (1998), Mulvey et al. (2000), Dempster et al. (2003), Geyer and Ziemba (2008), Dupačová and Polívka (2009) and de Oliveira et al. (2017).

Different approaches for modelling risk in the context of ALM can be found in the literature. They mainly stem from the single period asset allocation modelling framework, where the most common approach is to find investment decisions which result in a return distribution with a high expected value and low value of risk. Risk can be defined in a variety of ways depending on which unfavorable aspect of the return distribution is to be penalised. Decisions are then found as optimal solutions in models where expected value is maximised with a constraint on risk; alternatively, risk can be minimised with a constraint on expected value.

This modelling approach has been extended to the case of multi-period setting, liability driven investment by maximising the expected terminal fund wealth while imposing risk constraints in the intermediate time periods. When modelling risk in an ALM context, the distribution of interest is not necessarily that of wealth or asset return, as the relationship to liabilities is crucial. Instead, the distribution of interest is usually related to the funding ratio. Usually, a target funding ratio is set, a number $\lambda \geq 1$ below which the value of the funding ratio is desired not to fall. Other said, the undesirable situations are those in which the asset value falls below the liability value (multiplied by $\lambda$ ).

Fishburn (1977) used Lower Partial Moments (LPMs) in the context of single stage asset allocation models. The LPM with target $\tau$ and order $n$ of a random variable $R$ (e.g. representing future return) is by definition $E\left[\max \{\tau-R, 0\}^{n}\right]$. In the particular case $n=0$, the LPM measures the probability of falling below target $\tau$, which had been used in asset allocation models by Roy (1952).

For multi-stage ALM models, Dert (1995) proposed a similar approach in controlling the risk of underfunding, using the stochastic programming paradigm of chance constraint programming (Charnes and Cooper 1959). Chance constraints were implemented recently in a multistage SP for the Brazilian pension fund industry in de Oliveira et al. (2017). Omitting the time index, denote by $A$ the distribution of asset value and by $L$ the distribution of liabilities. The constraint $A \geq \lambda L$ under all scenarios is relaxed by allowing a small percentage of scenarios $\beta=B \%$ under which underfunding may happen. Formally, $\operatorname{Prob}(A-\lambda L<0) \leq \beta$ or equivalently $\operatorname{Prob}(A / L<\lambda) \leq \beta$. This is the same with imposing an upper limit $\beta$ on the lower partial moment with target $\lambda$ and order 0 of the random variable $A / L$ representing the funding ratio.

Chance constraints control the probability of constraint violation but do not account for the amount by which it is violated. In addition, they require binary variables to be 
implemented, thus increasing computational complexity. An alternative SP approach is the Integrated Chance Constraint Programming (ICCP), proposed by Klein Haneveld (1986) and Klein Haneveld and Van Der Vlerk (2006), used in the context of ALM by Klein Haneveld et al. (2010). With the above notations, an ICCP constraint requires that $E[\max \{\lambda L-A, 0\}] \leq \theta$, where $\theta$ is the maximum amount of average underfunding that a decision maker accepts. This is the same with imposing an upper limit $\theta$ on the lower partial moment with target 0 and order 1 of the random variable $A-\lambda L$.

Risk control in ALM models via Lower partial moments of order two has been adopted by Kouwenberg (2001): $E\left([\max \{\lambda L-A, 0\}]^{2}\right) \leq \theta$.

Conditional Value-at-Risk $(\mathrm{CVaR})$ was proposed by Rockafellar and Uryasev (2000) in the context of single stage asset allocation. Let a random variable (representing loss) and a number $\alpha=A \%$ representing a percentage of worst case outcomes. CVaR at confidence level $(1-\alpha)$ measures, largely speaking, "the average of losses in the worst A\% of cases". In the context of multi-stage ALM, it was used by Bogentoft et al. (2001); they considered the loss variable $\lambda L-A$ and imposed an upper limit on its CVaR.

The approaches above are thus related to the mean-risk paradigm, often employed in asset allocation due to its intuitive setting and, in most cases, computational simplicity. On the other hand, in imposing risk constraints, one single aspect of the distribution of interest is controlled. For example, a limit on the expected shortfall does not guarantee manageable worst case realisations and hence does not exclude the possibility of catastrophic losses; a distribution might have a very small percentage of very low outcomes and high outcomes in the rest; in this case, an integrated chance constraints may be satisfied but the worst case realisation could be unacceptably low. Similarly, a CVaR upper limit may guarantee manageable outcomes even under worst case scenarios, but it may leave open the possibility of under-achievement in the rest of the distribution. For instance, a distribution may be "flat" in that the worst case realisations are not very low, but with little improvement in the rest of the distribution.

In this paper, we propose an alternative way of controlling the risk of underfunding, using the concept of Second Order Stochastic Dominance (SSD). This is a criterion of ranking random variables that takes the entire distribution of outcomes into account. It is applied to random variables whose outcomes are desired to be high and for whom an increase is more valued if it is at low levels, rather than at high levels; typical such random variables represent return or wealth. SSD eliminates the need to specify a utility function but works under the general and widely accepted assumptions of decision makers being rational (utility function is non-decreasing) and risk averse (utility function is concave). By definition, a random variable is preferred to another with respect to SSD if its expected utility is higher, for any non-decreasing and concave utility function. It is obviously desirable to eliminate the random variables that are dominated and make a choice among the SSD non-dominated ones, possibly employing another criterion to help in the final selection.

The conceptual advantages of using SSD in asset allocation has been long recognised, along with computational difficulties in applying it (Whitmore and Findlay 1978). Recently, computationally tractable optimisation models based on SSD have been proposed for single stage portfolio optimisation-for example, Dentcheva and Ruszczynski (2006), Roman et al. (2006), Fábián et al. (2011a,b), Post and Kopa 
(2013), Kopa and Post (2015) and very recently for multistage SP problems-see for example Escudero et al. (2016) and Kopa et al. (2018).

In the optimisation models proposed in this paper, decision variables represent portfolio rebalancing decisions. SSD is used as a choice criterion for random variables representing funding ratios. The approaches developed in Roman et al. (2006) and Fábián et al. (2011b) are extended and adapted to the ALM multi-period case, taking into account the relationship between asset value and liabilities. An optimal solution has a corresponding funding ratio distribution that is SSD non-dominated; in addition, it comes close, in well defined sense, to a target distribution of funding ratio, whose outcomes are specified by the decision maker. Different target distributions lead to different SSD efficient solutions. A constraint on the expected terminal wealth is imposed, by considering a minimum acceptable compounded return. Improved distributions of funding ratios may be thus achieved, compared to the existing risk models for ALM. This represents the first contribution of the paper. The second contribution is of theoretical nature; interesting results, connecting the proposed models to well established risk models and well established classes of SP models are derived for the particular case when the target distribution is deterministic, specified by one single outcome.

Previously, SSD has been used in multi-stage ALM models by Yang et al. (2010), by imposing a stochastic dominance constraint: the objective is the maximise expected terminal wealth, while the distribution of asset value is constrained to dominate, with respect to SSD, a given benchmark distribution. More recently, Kopa et al. (2018) apply first and second order stochastic dominance constraints in a multistage SP model for individual optimal pension allocation. The objective is to minimize the Average Value at Risk Deviation measure while satisfying a wealth target; the optimum portfolio is constrained to dominate a benchmark with respect to stochastic dominance relations. In our approach, the the SSD criterion is applied to funding ratio distributions and in the objective, rather than as a contraint. This overcomes undesirable situations that might occur, depending on the benchmark distribution chosen by the decision maker. For example, if the outcomes of the benchmark distribution are too high and as a result this distribution cannot be dominated or attained, a stochastic dominance constraint would result in infeasibility. In the opposite situation when the benchmark distribution is SSD dominated, the optimal solution is guaranteed to improve on the benchamark but not necessarily to be SSD non-dominated.

With the family of models proposed in this paper, there are three possible cases. Firstly, if the benchmark distribution is dominated with respect to SSD, the optimal solutionl results in a funding ratio distribution which is "better than target": it improves on the benchmark until SSD efficiency is attained. Secondly, if the benchmark is SSD efficient, the optimal solution of the model has a funding ratio distribution that exactly matches the benchmark. Finally, if the target is not attainable (in the sense that no feasible solution could match or improve on it), the optimal solution has a funding ratio distribution which is SSD efficient and comes as close as possible, in a well defined sense, to the target.

The rest of this paper is organised as follow. In the next section we set a basic framework for an ALM decision problem and recall main risk modelling frameworks: chance constraint programming, integrated chance constraint programming, Conditional Value-at Risk. Section 3 presents the mathematical formulation of the proposed SSD 
models; a benchmark distribution of funding ratio is assumed to be available. Section 4 presents the connection with the ICCP formulation and more generally, the meanrisk framework, for the particular case when the benchmark distribution is degenerate. A numerical experiment is presented in Sect. 5, using a dataset drawn from a large defined benefit pension fund in Saudi Arabia. Conclusions are presented in Sect. 6.

\section{ALM problem setting}

We consider a pension fund problem in which the planning horizon is split into $T$ sub-periods. At each time point $t \in\{0, \ldots, T-1\}$ a decision is made on rebalancing the portfolio allocations. At each of these time points, liabilities are to be paid out and contributions are to be paid in.

The returns of the available assets, as well as values of liabilities and contributions are observed at times $t \in\{1, \ldots, T\}$. The uncertainty about asset returns, liabilities and contributions is modelled by a set of $S$ sample paths, or scenarios. Each scenario has an associated probability of occurrence $\pi_{s}, \forall s \in\{1, \ldots, S\}$, where $\pi_{s}>0$ and $\sum_{s=1}^{S} \pi_{s}=1$. Similarly to Bogentoft et al. (2001), we consider a scenario tree in the form of a fan; the root node represents the present $(t=0)$ when first stage decisions about portfolio rebalancing need to be taken. Each path from $t=0$ to $t=T$ represents one scenario, that is, one possible sequence of outcomes of the stochastic elements throughout the time horizon under consideration. The fund's total value of assets is evaluated at time $T$; in the intermediate time periods, risk constraints are imposed, usually related to the funding ratio not being lower than a pre-specified target level $\lambda \geq 1$. Values of $\lambda>1$ often are used to add some extra safety margin.

In what follows, we present the basic modelling framework. We use the following notations:

$I=$ number of financial assets available for investment

$T=$ number of time periods

$S=$ number of scenarios

The parameters of the model are denoted by:

$L_{t, s}=$ Liability value for time period $\mathrm{t}$ under scenario $\mathrm{s} ; t=1 \ldots T, s=1 \ldots S$

$C_{t, s}=$ The contributions paid into the fund at time period $t$ under scenario $s$; $t=1 \ldots T, s=1 \ldots S$

$R_{i, t, s}=$ The rate of return of asset $i$ at time period $t$ under scenario $s ; i=1 \ldots I$, $t=1 \ldots T, s=1 \ldots S$

$\pi_{s}=$ The probability of scenario $s$ occurring; $s=1 \ldots S$

$O P_{i}=$ The amount of money held in asset $i$ at the initial time period $t=0$; $i=1 \ldots I$

$\psi=$ The transaction cost expressed as a percentage of the value of each trade

$L_{0}=$ Aggregated liability payments to be made "now" $(t=0)$

$C_{0}=$ The funding contributions received "now" $(t=0)$

Let us denote the first stage decision variables by:

$B_{i, 0}=$ The monetary value of asset $i$ bought at the beginning of the planning horizon $(t=0) ; i=1 \ldots I$ 
$S_{i, 0}=$ The monetary value of asset $i$ sold at $t=0 ; i=1 \ldots I$

$H_{i, 0}=$ The monetary value of asset $i$ held at $t=0 ; i=1 \ldots I$

with $H_{i, 0}=O P_{i}+B_{i, 0}-S_{i, 0}, \quad i=1 \ldots I$.

Let us denote the recourse decision variables by:

$H_{i, t, s}=$ The monetary value of asset $i$ held at time $t$ under scenario $s ; i=1 \ldots I$, $t=1 \ldots T, s=1 \ldots S$

$B_{i, t, s}=$ The monetary value of asset $i$ bought at time $t$ under scenario $s ; i=1 \ldots I$, $t=1 \ldots T-1, s=1 \ldots S$

$S_{i, t, s}=$ The monetary value of asset $i$ sold at time $t$ under scenario $s ; i=1 \ldots I$, $t=1 \ldots T-1, s=1 \ldots S$

Denote by $A_{t, s}$ the total asset value at time $t$ under scenario $s$, before portfolio rebalancing. The following relations hold:

$$
\begin{aligned}
& A_{t, s}=\sum_{i=1}^{I} H_{i, t-1, s} R_{i, t, s}, \quad t=2 \ldots T-1, s=1 \ldots S \\
& A_{1, s}=\sum_{i=1}^{I} H_{i, 0} R_{i, 1, s}, \quad s=1 \ldots S
\end{aligned}
$$

At each time point $t \in\{1, \ldots, T\}$, the asset value $A_{t}$ and the liability value $L_{t}$ are random variables with outcomes $\left\{A_{t, s}\right\}_{s=1 \ldots S}$ and $\left\{L_{t, s}\right\}_{s=1 \ldots S}$, occurring with probabilities $\left\{\pi_{s}\right\}_{s=1 \ldots S}$.

The common approach encountered in the literature is to maximise the expected value of the terminal asset value $A_{T}$ while imposing risk constraints on short or medium term. The funding ratio at time t, denoted here by $F_{t}$ and defined as $A_{t} / L_{t}$ is commonly used to model risk constraints, although there are variations in the way these are modelled. Ideally, we would like $A_{t} \geq \lambda L_{t}$, or $F_{t} \geq \lambda$ with probability 1 ; this however might be impossible and/or very costly for the long term wealth of the fund.

Dert (1995) used a chance constraint, imposing instead $A_{t} \geq \lambda L_{t}$ with high (prespecified) probability. Such a constraint is modelled using $S$ additional binary variables for every time period when a chance constraint is imposed. The binary variables count the number of times the constraint is violated; $\delta_{t, s}=1$ when $A_{t, s}<\lambda L_{t, s}$ and 0 otherwise.

Chance constraints have no control on the amount of shortfall; there is the possibility of the amount of underfunding (occurring under low probability) being unacceptably large. Klein Haneveld et al. (2010) apply integrated chance constraints (ICCs) for modelling short term risk $(t=1)$ in an ALM model for Dutch pension funds. With an ICC, the amount of expected shortfall is controlled, rather than the probability of shortfall: $E\left[\max \left\{\lambda L_{t}-A_{t}, 0\right\}\right] \leq \theta$, where $\theta$ is the maximum amount of average underfunding that a decision maker accepts. This could be equivalently formulated as $E\left[\max \left\{\lambda-F_{t}, 0\right\}\right]$ does not exceed a pre-specified level, hence imposing an upper bound on the lower partial moment of order 1 and target $\lambda$ of the funding ratio. Modelling such a constraint does not require additional binary variables but only continuous ones; we formulate this in Sect. 4.2. 
Bogentoft et al. (2001) considered a loss random variable defined by $\lambda L_{t}-A_{t}$ and imposed a CVaR constraint for short term risk $(\mathrm{t}=1)$. If CVaR is considered at confidence level $A \%=\alpha \in(0,1)($ e.g. $\alpha=0.95)$ then this constraint can be expressed as follows: the average of the highest $(1-A) \%$ outcomes of the loss is no higher than a level pre-specified by the decision-maker. Just as with ICC constraints, a CVaR constraint is modelled by introducing additional (continuous) variables and linear constraints; the reader is referred to Rockafellar and Uryasev (2000) and Bogentoft et al. (2001).

We can summarise the difference between an ICC constraint and a CVaR constraint as follows. With ICC constraints, all the outcomes of the funding ratio are considered, in which the target funding ratio is not met. With a CVaR constraint, the percentage of worst case outcomes is fixed in advance; these worst case outcomes may or may not include all the cases in which the target funding ratio is not met. It can be argued that ICC provides a better modelling approach since all scenarios when underfunding occurs are considered. On the other hand, there is less control in worst case scenarios; although the average underfunding may seem acceptable, the possibility exists for a heavy left tailed loss distribution. This leaves the open question on what risk measure is most appropriate.

An ALM model with a generic risk constraint can be formulated as follows:

$$
\max \sum_{s=1}^{S} \pi_{S} A_{T, s}
$$

subject to:

$$
\begin{aligned}
& H_{i, 0}=O P_{i}+B_{i, 0}-S_{i, 0}, \quad i=1 \ldots I \\
& H_{i, 1, s}=H_{i, 0} R_{i, 1, s}+B_{i, 1, s}-S_{i, 1, s}, \quad i=1 \ldots I, \quad s=1 \ldots S \\
& H_{i, t, s}=H_{i, t-1, s} R_{i, t, s}+B_{i, t, s}-S_{i, t, s}, \quad i=1 \ldots I, t=2 \ldots T-1, \\
& \quad s=1 \ldots S \\
& H_{i, T, s}=H_{i, T-1, s} R_{i, T, s}, \quad i=1 \ldots I, s=1 \ldots S \\
& \sum_{i=1}^{I} B_{i, 0}(1+\psi)+L_{0}=\sum_{i=1}^{I} S_{i, 0}(1-\psi)+C_{0} \\
& \sum_{i=1}^{I} B_{i, t, s}(1+\psi)+L_{t, s}=C_{t, s}+\sum_{i=1}^{I} S_{i, t, s}(1-\psi), \quad t=1 \ldots T-1, \\
& \quad s=1 \ldots S \\
& A_{1, s}=\sum_{i=1}^{I} H_{i, 0} R_{i, 1, s}, \quad s=1 \ldots S \\
& A_{t, s}=\sum_{i=1}^{I} H_{i, t-1, s} R_{i, t, s}, \quad t=2 \ldots T, s=1 \ldots S
\end{aligned}
$$




$$
\begin{aligned}
& B_{i, 0}, S_{i, 0}, H_{i, 0}, B_{i, t, s}, S_{i, t, s}, H_{i, t, s}, H_{i, T, s} \geq 0, \quad i=1 \ldots I, t=1 \ldots T-1, \\
& \quad s=1 \ldots S \\
& \rho\left(A_{t}-\lambda L_{t}\right) \leq \theta
\end{aligned}
$$

Equations (6) and (7) are cash balance constraints. Equation (11) expresses a risk constraint with a generic risk measure $\rho$ of the random variable $A_{t}-\lambda L_{t}$; the risk value must not exceed a user pre-specified level $\theta$. A common approach is to impose a risk constraint for $\mathrm{t}=1$ (as per Klein Haneveld et al. 2010; Bogentoft et al. 2001); this can be generalised to include other time periods.

We propose an alternative risk modelling framework based on the concept of Second Order Stochastic Dominance (SSD).

\section{Second order stochastic dominance in ALM models}

SSD is a preference relation among random variables-representing, for example, portfolio returns, or asset values, or funding ratios_-defined by the following equivalent conditions:

(a) $\mathrm{E}(U(R)) \geq \mathrm{E}\left(U\left(R^{\prime}\right)\right)$ holds for any utility function $U$ that has the properties of non-satiation (it is non-decreasing, first derivative is positive) and risk aversion (it is concave, second derivative is negative) and for which these expected values exist and are finite.

(b) $\mathrm{E}\left([\tau-R]_{+}\right) \leq \mathrm{E}\left(\left[\tau-R^{\prime}\right]_{+}\right)$holds for each target $\tau \in \mathbb{R}$. Other said, the expected shortfall with respect to any target is always lower for the first random variable.

(c) $\operatorname{Tail}_{\alpha}(R) \geq \operatorname{Tail}_{\alpha}\left(R^{\prime}\right)$ holds for each $0<\alpha \leq 1$, where $\operatorname{Tail}_{\alpha}(R)$ denotes the unconditional expectation of the lowest $\alpha * 100 \%$ of the outcomes of $R$.

(d) $\operatorname{Scaled~Tail~}_{\alpha}(R) \geq \operatorname{Scaled~Tail}_{\alpha}\left(R^{\prime}\right)$ holds for each $0<\alpha \leq 1$, where $\operatorname{ScaledTail}_{\alpha}(R)$ denotes the conditional expectation of the lowest $\alpha * 100 \%$ of

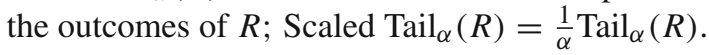

If the relations above hold, the random variable $R$ is said to dominate the random variable $R^{\prime}$ with respect to SSD.

The equivalence of the above relations was proved in Whitmore and Findlay (1978) and Ogryczak and Ruszczynski (2002); please see also Fábián et al. (2011a).

Remark 1 The definitions of $\operatorname{Tail}_{\alpha}(R)$ and $\operatorname{ScaledTail}_{\alpha}(R)$ are informal. For formal definitions, quantile functions can be used. Denote by $F_{R}$ the cumulative distribution function of a random variable $R$. If there exists $t$ such that $F_{R}(t)=\alpha$ then $\operatorname{Tail}_{\alpha}(R)=$ $\alpha E(R \mid R \leq t)$ and $\operatorname{Scaled~Tail}_{\alpha}(R)=E(R \mid R \leq t)$-which justifies the informal definitions.

For the general case, let us define the generalised inverse of $F_{R}$ as $F_{R}^{-1}(\alpha):=$ $\inf \left\{t \mid F_{R}(t) \geq \alpha\right\}$ and the second quantile function as $F_{R}^{-2}(\alpha):=\int_{0}^{\alpha} F_{R}^{-1}(\beta) d \beta$ and $F_{R}^{-2}(0):=0$. Then, $\operatorname{Tail}_{\alpha}(R):=F_{R}^{-2}(\alpha)$. 
The relationship with Conditional Value at Risk (CVaR) has been discussed in the literature in Kopa and Chovanec (2008) and later on in Fábián et al. (2011a). Assuming loss is described by the random variable $-R$, we have:

$\operatorname{Tail}_{\alpha}(R):=-\alpha \operatorname{CVaR}_{1-\alpha}(-R)$ and $\operatorname{ScaledTail}_{\alpha}(R):=-\mathrm{CVaR}_{1-\alpha}(-R)$.

Remark 2 The relation in point (b) shows the connection between LPM of order 1 and $\mathrm{SSD} ; R$ dominates $R^{\prime}$ with respect to second order stochastic dominance if the LPM of order 1 and target $\tau$ of $R$ is smaller than LPM of order 1 of $R^{\prime}$ for all targets $\tau \in \mathbb{R}$. This is a particular case of a more general relationship between stochastic dominance and LPMs shown in Post and Kopa (2013).

The importance of SSD in financial asset allocation can be clearly seen: it expresses the preference of rational and risk-averse decision makers.

We propose an ALM model in which the first stage investment decisions are such that the resulting funding ratio distribution is non-dominated with respect to SSD, or in other words, SSD efficient. Similarly to Klein Haneveld et al. (2010), we consider the funding ratio at time $t=1$, thus modelling short term risk; the approach can be extended for more time periods.

Following Roman et al. (2006), we assume that the probabilities of scenarios are equal, that is $\pi_{s}=1 / S, s=1 \ldots S$. This is the usual situation when scenarios are generated via simulation or sampled from historical data. In this case, as shown in Roman et al. (2006), Kopa and Chovanec (2008) also used in Fábián et al. (2011a,b), the comparison with respect to SSD can be greatly simplified: for example, in relations (c) and (d) from the definition of SSD, it is enough to consider $\alpha$ only in the finite set $\{1 / S, 2 / S, \ldots, S / S\}$ rather than in the interval $(0,1)$. Other said, it is enough to compare tails only for confidence levels $\frac{k}{S}$ with $k=1 \ldots S$.

Let us consider two sets of first stage decisions $\left(H_{i, 0}, i=1 \ldots I\right)$ and $\left(H_{i, 0}^{\prime}, i=\right.$ $1 \ldots I$ ) with corresponding funding ratios $F$ and $F^{\prime}$ respectively, having as possible outcomes

$$
F_{s}=\sum_{i=1}^{I} H_{i, 0} R_{i, 1, s} / L_{1, s}, \quad s=1 \ldots S
$$

and

$$
F_{s}^{\prime}=\sum_{i=1}^{I} H_{i, 0}^{\prime} R_{i, 1, s} / L_{1, s}, \quad s=1 \ldots S
$$

respectively; each of these outcomes occurs with probability $1 / S$.

Let us order $F_{1}, \ldots F_{S}$ and $F_{1}^{\prime}, \ldots F_{S}^{\prime}$ and lets us denote by $\quad \alpha_{1} \leq \ldots \leq \alpha_{S}$ the outcomes of $F$ in ascending order and $\beta_{1} \leq \ldots \leq \beta_{S}$ the outcomes of $F^{\prime}$ in ascending order. It is clear that:

$$
\operatorname{Tail}_{k / S}(F)=\left(\sum_{i=1}^{k} \alpha_{i}\right) / S \text { and } \operatorname{ScaledTail}_{k / S}(F)=\left(\sum_{i=1}^{k} \alpha_{i}\right) / k \text {. }
$$

With the notations here, the relationships developed in Roman et al. (2006) can be written as: 
$F$ dominates $F^{\prime}$ with respect to SSD if and only if

$$
\operatorname{Tail}_{k / S}(F) \geq \operatorname{Tail}_{k / S}\left(F^{\prime}\right), \quad k=1 \ldots S
$$

or equivalently,

$$
\operatorname{ScaledTail}_{k / S}(F) \geq \operatorname{ScaledTail}_{k / S}\left(F^{\prime}\right), \quad k=1 \ldots S
$$

with at least one strict inequality; please also see Kopa and Chovanec (2008).

Thus, the SSD efficient solutions are the Pareto non-dominated optimal solutions in a multi-objective optimisation problem in which the objective functions to maximise are the tails (or scaled tails) of the funding ratio distribution $F$, at confidence levels $\frac{k}{S}$, with $k=1 \ldots S$ :

$$
\operatorname{Max}\left(\operatorname{Tail}_{1 / S}(F), \operatorname{Tail}_{2 / S}(F), \ldots, \operatorname{Tail}_{S / S}(F)\right)
$$

or we can use the "scaled" version:

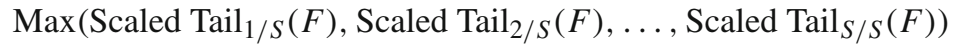

subject to: (2)-(10).

Remark 3 Due to the relationship between CVaR and scaled tails expresed in Remark 1 , it can be clearly seen that (13) is equivalent to a multi-objective minimisation model in which the objective functions are CVaRs at confidence levels $\left(1-\frac{1}{S}, 1-\frac{2}{S}, \ldots, 0\right)$.

There are various methods of obtaining a Pareto optimal solution of a multiobjective optimisation problem. A good control on obtaining a specific solution is given by the Reference Point Method (Wierzbicki 1982), where target points (also called reference points) are set for each objective function. Consider the general case of $S$ realvalued objective functions $F_{1}, \ldots F_{S}$ defined on a set $X \in \mathbb{R}^{n}$ representing a feasible set of decision vectors and consider the multi-objective model: $\operatorname{Max}\left(F_{1}(x), \ldots F_{S}(x)\right)$ s.t. $x \in X$. For each of the functions $F_{k}$ a target point $f_{k}^{*}$ is set by the decision maker; partial achievements $\left(F_{k}(x)-f_{k}^{*}\right), k=1 \ldots S$ can be measured for any feasible point. In Wierzbicki (1982), it is shown that maximisation of the worst partial achievement, that is, maximisation of $\operatorname{Min}\left\{F_{1}(x)-f_{1}^{*}, \ldots, F_{S}(x)-f_{S}^{*}\right\}$ results in a Pareto optimal solution of the multi-objective model, apart possibly from the case of multiple optimal solutions. To guarantee Pareto efficiency in the general case, a regularization term is added to the worst partial achievement: $\epsilon \sum_{k=1}^{S}\left(F_{k}(x)-f_{k}^{*}\right)$, where $\epsilon>0$. It was shown in Wierzbicki (1982) a Pareto optimal solution of the multi-objective model is obtained for any $\epsilon>0$; however, a small enough $\epsilon$ should be chosen (possibly on a trail and error basis) if optimisation of the worst partial achievement is desired.

Similarly to Roman et al. (2006), we use the Reference Point Method in order to obtain Pareto optimal solutions of (13), that is, SSD efficient solutions in the ALM model. In our case, the objective functions represent tails or scaled tails (at different confidence levels) of the funding ratio distribution. Thus, the target points for each objective function define a target, or "reference" distribution of funding ratio. 
Let us consider a target distribution of the funding ratio, with (equally probable) outcomes $\lambda_{k}, k=1 \ldots S$; without loss of generality, let us consider $\lambda_{1} \leq \ldots \leq \lambda_{S}$.

Let us denote by $a s p_{k}$ the scaled $k$ th cumulative outcome,

$$
\operatorname{asp}_{k}=\frac{1}{k} \sum_{i=1}^{k} \lambda_{i}, \quad k=1 \ldots S
$$

and by $a s p_{k}^{\prime}$ be the unscaled $k$ th cumulative outcome,

$$
\operatorname{asp}_{k}^{\prime}=\frac{1}{S} \sum_{i=1}^{k} \lambda_{i}, \quad k=1 \ldots S
$$

The target point for the $k$ th objective function in (13) is $a s p_{k}$, while $a s p_{k}^{\prime}$ represents the target point for the $k$ th objective function in (14). Following Wierzbicki (1982), the multi-objective model (13) is transformed into a single objective model by maximising the following achievement function:

$$
\min _{k=1 \ldots S}\left(\operatorname{Scaled~Tail~}_{k / S}(F)-\operatorname{asp}_{k}\right)+\epsilon \sum_{k=1}^{S}\left(\operatorname{Scaled~Tail~}_{k / S}(F)-\operatorname{asp}_{k}\right)
$$

If the unscaled model is used, the objective function to maximise is:

$$
\min _{k=1 \ldots S}\left(\operatorname{Tail}_{k / S}(F)-\operatorname{asp}_{k}^{\prime}\right)+\epsilon \sum_{k=1}^{S}\left(\operatorname{Tail}_{k / S}(F)-\operatorname{asp}_{k}^{\prime}\right)
$$

In order to express the tails and scaled tails of the funding ratio distribution as functions of the decision variables, we adopt the CVaR formulation of Rockafellar and Uryasev in Rockafellar and Uryasev (2000). It expresses a cumulative outcome of a random variable as the optimal value of an LP model:

Proposition 1 For every $k \in\{1, \ldots, S\}$, the mean of the worst $k$ outcomes of a random variable $\boldsymbol{y}$ with equally likely outcomes $y_{1}, \ldots, y_{S}$ is the optimal value of the objective function in the following LP problem:

$$
\operatorname{Max}\left[T_{k}-\frac{1}{k} \sum_{i=1}^{S} d_{k, i}\right]
$$

\section{Subject to:}

$$
\begin{aligned}
T_{k}-y_{s} & \leq d_{k, s}, \quad s=1 \ldots S \\
d_{k, s} & \geq 0, \quad k, s=1 \ldots S
\end{aligned}
$$

$T_{k}$ is a free variable representing the $k$ th worst outcome of the random variable $y$. 
For each $s \in\{1, \ldots, S\}, d_{k, s}$ takes value 0 if $y_{s}$ is greater than or equal to the $k$ th worst outcome $T_{k}$; otherwise, $d_{k, s}=T_{k}-y_{s}$.

For proof, the reader is referred to Rockafellar and Uryasev (2000).

Hence, (13) becomes:

$$
\operatorname{Max}\left(T_{1}-\sum_{i=1}^{S} d_{1, i}, T_{2}-\frac{1}{2} \sum_{i=1}^{S} d_{2, i}, \ldots, T_{S}-\frac{1}{S} \sum_{i=1}^{S} d_{S, i}\right)
$$

\section{Subject to:}

$$
\begin{aligned}
T_{k}-F_{s} & \leq d_{k, s}, \quad k, s=1 \ldots S \\
d_{k, s} & \geq 0, \quad k, s=1 \ldots S
\end{aligned}
$$

Thus, the following SSD scaled model is formulated:

$$
\operatorname{Max} \delta+\epsilon\left(\sum_{k=1}^{S} Z_{k}-\sum_{k=1}^{S} a s p_{k}\right)
$$

\section{Subject to:}

$$
\begin{aligned}
& Z_{k}=T_{k}-\frac{1}{k} \sum_{s=1}^{S} d_{k, s}, \quad k=1 \ldots S \\
& Z_{k}-\operatorname{asp}_{k} \geq \delta, \quad k=1 \ldots S \\
& T_{k}-F_{s} \leq d_{k, s}, \quad k, s=1 \ldots S \\
& F_{s}=\sum_{i=1}^{I} H_{i, 0} R_{i, 1, s} / L_{1, s} \quad\left(F_{S}=A_{1, s} / L_{1, s}\right), \quad s=1 \ldots S \\
& d_{k, s} \geq 0, \quad k, s=1 \ldots S \\
& \frac{1}{S} \sum_{s=1}^{S} A_{T, s} \geq \sum_{i=1}^{I} O P_{i}(1+r)
\end{aligned}
$$

and also subject to (2)-(10).

In addition to the decision variables $H_{i, 0}, B_{i, 0}, S_{i, 0}, H_{i, t, s}, B_{i, t, s}, S_{i, t, s}$ representing investment decisions, we have additional decision variables whose nature is discussed below:

$T_{k}=$ the $k$ th worst outcome of the funding ratio at time $1, \mathrm{k}=1 \ldots \mathrm{S}$ (free variables); thus, $T_{1}, \ldots, T_{S}$ are the outcomes of a random variable equal in distribution to the funding ratio;

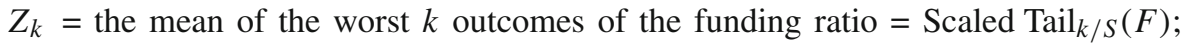
$Z_{k}=\left(T_{1}+\ldots+T_{k}\right) / k, k=1 \ldots S$ (free variables); 
$\delta=\min _{k=1 \ldots S}\left(Z_{k}-a s p_{k}\right)=$ the worst partial achievement (free variable);

$d_{k, s}$ are non-negative variables; $d_{k, s}=0$ if the funding ratio under scenario $s$ is greater than or equal to the $k$ th worst funding ratio $T_{k}$; otherwise, $d_{k, s}$ is the difference between $T_{k}$ and the funding ratio $F_{s} ; k, s=1 \ldots S$.

In addition to the parameters already discussed representing returns of the assets, liabilities, contributions and initial portfolio, there are parameters which are chosen by the decision maker:

$\operatorname{asp}_{k}=$ the target or aspiration level for $Z_{k}=\operatorname{Scaled~Tail}_{k / S}(F), k=1 \ldots S$;

$r>0=$ desired rate of return over the investment horizon;

$\epsilon>0=$ the weighting coefficient of the regularisation term in the objective function.

For any choice of aspiration levels and of $\epsilon>0$, the optimal solution of the above model represents a first stage decision allocation $H_{i 0}, i=1 \ldots$ A such that the corresponding funding ratio $F$, represented by equally probable outcomes $F_{s}, s=$ $1 \ldots S$, is SSD efficient.

To prove this, let us suppose that this is not true and the funding ratio $F$ is $\mathrm{SSD}$ dominated; this means that there is another feasible decision $H_{i 0}^{*}, i=$ $1 \ldots A$ with a corresponding funding ratio distribution $F^{*}$ (denote its outcomes by $F_{s}^{*}, s=1 \ldots S$ ) that dominates F with respect to SSD. That is, $\operatorname{Scaled} \operatorname{Tail}_{k / S}\left(F^{*}\right) \geq$ Scaled Tail $_{k / S}(F), \quad k=1 \ldots S$ with at least one inequality strict, or with notations above $Z_{k}^{*} \geq Z_{k}, \quad k=1 \ldots S$ with at least one inequality strict, where $Z_{k}^{*}=\operatorname{Scaled~Tail~}_{k / S}\left(F^{*}\right), k=1 \ldots S$. Denote by $\delta^{*}=\min _{k=1 \ldots S}\left(Z_{k}^{*}-\operatorname{asp}_{k}\right)$. It follows that $\delta^{*}+\epsilon\left(\sum_{k=1}^{S} Z_{k}^{*}-\sum_{k=1}^{S} a s p_{k}\right)>\delta+\epsilon\left(\sum_{k=1}^{S} Z_{k}-\sum_{k=1}^{S} a s p_{k}\right)$. For each $k \in\{1, \ldots, S\}$, we solve the model:

$$
\operatorname{Max}\left[T_{k}-\frac{1}{k} \sum_{i=1}^{S} d_{k, i}\right]
$$

\section{Subject to:}

$$
\begin{aligned}
T_{k}-F_{s}^{*} & \leq d_{k, s}, \quad s=1 \ldots S \\
d_{k, s} & \geq 0, \quad k, s=1 \ldots S
\end{aligned}
$$

and denote by $T_{k}^{*}, d_{k, s}^{*}, s=1 \ldots S$ the optimal solution. We obtain thus a feasible solution for the (SSD scaled) model that results in a strictly better value of the objective function, which is a contradiction.

Remark 4 The sign of the optimal value of $\delta$ or of the optimal value of the objective function in (SSD scaled) is an indication of whether the aspiration levels have been achieved. A strictly positive $\delta$ indicates that the aspiration levels have been strictly improved upon. If the aspiration distribution is exactly matched, that is, if the optimal solution results in a funding ratio distribution whose scaled tails are exactly the reference points, the optimum value of the objective function is 0 . Finally, a strictly negative optimum indicates that there is at least one scaled tail that did not achieve its target. 
By considering unscaled tails in the multi-objective optimisation, we obtain the SSD unscaled model:

$$
\operatorname{Max} \delta^{\prime}+\epsilon\left(\sum_{k=1}^{S} Z_{k}^{\prime}-\sum_{k=1}^{S} a s p_{k}^{\prime}\right)
$$

\section{Subject to:}

$$
\begin{aligned}
& Z_{k}^{\prime}=k T_{k}-\sum_{s=1}^{S} d_{k, s}, \quad k=1 \ldots S \\
& Z_{k}^{\prime}-a s p_{k}^{\prime} \geq \delta^{\prime}, \quad k=1 \ldots S
\end{aligned}
$$

also subject to (2)-(10) and to (16)-(19).

Remark 5 Both (SSD scaled) and (SSD unscaled) models have (possibly different) optimal solutions such that their distribution of funding ratio is SSD efficient. The modelling difference resides in how the "closeness" to the target distribution is measured, more precisely, how the shortfalls below target points are penalised. With the unscaled model, the accumulation of outcomes below their targets is penalised, rather than the magnitude of the shortfalls, which is more severely penalised in the scaled model. This becomes more obvious when the target distribution is degenerate, having one single possible outcome. It is shown in the next section that, in this case, the scaled model maximises the worst outcome of the funding ratio, i.e. the largest deviation from the (single) target point, while the unscaled model minimises the expected shortfall below the target, taking thus into account all situations when the target is not achieved.

Remark 6 Both (SSD scaled) and (SSD unscaled) models provide an SSD efficient solution, irrespective of the aspiration levels chosen by the decision maker; this choice cannot lead to infeasibility either. This follows from the "better than target" property of the Reference Point Method in multi-objective optimisation. It was shown in Wierzbicki (1982) that the maximisation of the achievement function results in a Pareto optimal solution of the multi-objective model irrespective of the reference points chosen by the decision maker. If the reference points do not form a Pareto optimal vector for the multi-objective model, the maximisation of the achievement function improves on the reference points until Pareto optimality is attained. If the reference points form a Pareto optimal vector, the optimal solution in the maximisation of the achievement function results in objective function values equal to the reference points. Finally, if at least one of the reference points is unattainable/too high, we obtain a Pareto optimal solution in which the worst difference between objective function values and reference points is optimised.

In the current setting, the multiple objective functions represent tails (or scaled tails) of the funding ratio distribution and Pareto optimal solutions represent SSD efficient distributions. The three cases above relate to whether the target distribution of funding ratio is (1) SSD dominated; (2) SSD efficient or (3) unattainable, in the sense that 
there is no feasible solution that could attain or improve on all of its tails. In all three cases, the optimal solutions of both scaled and unscaled models are SSD efficient, corresponding to cases (1) better than target; (2) matching the target; (3) coming close to the target distribution.

\section{Connection with risk minimisation}

Consider the particular case in which the target level for every outcome of the funding ratio distribution is equal to target funding ratio $\lambda ; \lambda_{1}=\lambda_{2}=\ldots=\lambda_{S}=\lambda$. This makes the target distribution a degenerate, or deterministic distribution.

\subsection{The SSD scaled model}

In this case, the aspiration levels for the scaled tails of the funding ratio are also all equal to $\lambda: \operatorname{asp}_{k}=\lambda, \forall k \in\{1, \ldots, S\}$.

The worst partial achievement $\delta=\min _{k=1 \ldots S}\left(Z_{k}-a s p_{k}\right)$ corresponds to the worst outcome of the funding ratio distribution. Thus, maximising the worst partial achievement is equivalent to maximising the worst outcome. A minimax mean-risk model, in which risk is defined as the maximum possible loss, was proposed by Young (1998), who also showed that such a model can be formulated as an LP.

In our case a similar Maximin model can be formulated, which optimises the worst outcome of funding ratio, subject to a constraint on the expected terminal wealth.

\section{$\operatorname{Max} \delta$}

\section{Subject to:}

$$
F_{s} \geq \delta, \quad s=1 \ldots S
$$

also subject to (2)-(10), (17) and (19).

In case the model above has a unique optimal solution, SSD efficiency is guaranteed. However, just as with the general SSD model, in case of non-unique optimal solutions, the SSD efficiency is not guaranteed; a regularisation term should be added in the objective function. The regularisation term in the SSD scaled model is the sum of tails/cumulated outcomes; in order to formulate tails, we need additional $S^{2}$ variables $d_{k i}$ which adds substantially to the computational complexity. In order to avoid this, we can add in the objective function above a term such as $\epsilon \sum_{S=1}^{S} F_{S}$ which brings no extra computational complexity; we obtain the model Maximin 2:

$$
\operatorname{Max}\left(\delta+\epsilon \sum_{s=1}^{S} F_{S}\right)
$$

\section{Subject to:}

$$
F_{s} \geq \delta, \quad s=1 \ldots S
$$


also subject to (2)-(10), (17) and (19).

Just as before, $\epsilon$ has to be chosen as a small enough number such that the optimisation is basically that of the worst outcome. The optimal solution of this model will result in a funding ratio that has the highest "worst" outcome and also the highest expected value amongst all optimal solutions of (Maximin). Notice that, although the chance of getting an SSD inefficient solution is substantially decreased at no extra computational complexity, SSD efficiency is still not guaranteed as there is theoretically the possibility that (Maximin 2) has multiple optimal solutions.

Thus, an SSD scaled model in which the reference distribution is degenerate could be in most cases written as a maximin model of minimising worst case outcome. The single outcome of the reference distribution is irrelevant.

\subsection{The SSD unscaled model}

The aspiration levels for the cumulated outcomes of the funding ratio are: $a s p_{k}^{\prime}=\frac{1}{S} k \lambda$, $k=1 \ldots S$. As in Sect. 4.1, denote by $T_{1} \leq \ldots \leq T_{S}$ the ordered outcomes of the funding ratio. The worst partial achievement is:

$$
\frac{1}{S} \min _{k=1 \ldots S}\left(T_{1}+\ldots+T_{k}-k \lambda\right)
$$

As each outcome below $\lambda$ is penalised, the minimum is achieved for an index $j$ in $\{1 \ldots S\}$ such that $T_{j} \leq \lambda \leq T_{j+1}$.

The worst partial achievement is thus

$$
\frac{1}{S}\left[\left(T_{1}-\lambda\right)+\ldots+\left(T_{j}-\lambda\right)\right]=\frac{1}{S} \sum_{T_{k}<\lambda}\left(T_{k}-\lambda\right)
$$

Thus, maximising the worst partial achievement is equivalent to minimising

$$
\frac{1}{S} \sum_{T_{k}<\lambda}\left(\lambda-T_{k}\right)
$$

which is the Lower Partial Moment of order 1 and target $\lambda$ of the funding ratio, also called the expected shortfall below target $\lambda$.

The model that minimises the expected shortfall below target $\lambda$ can be formulated as an LP by introducing $S$ additional variables representing the shortage of the funding ratio with respect to target $\lambda$ under each scenario:

$$
\operatorname{Min} \frac{1}{S} \sum_{s=1}^{S} S h_{s}
$$

\section{Subject to:}

$$
\begin{aligned}
F_{s}-\lambda+S h_{s} & \geq 0, & s & =1 \ldots S \\
S h_{t, s} & \geq 0, & s & =1 \ldots S
\end{aligned}
$$


also subject to (2)-(10), (17) and (19).

We notice several things here.

First, without the addition of a regularisation term, minimisation of the expected shortfall is not guaranteed to result in an SSD efficient solution. One case in which SSD efficiency might not occur is the situation when there are multiple optimal solutions; at least one of them is SSD efficient but not necessarily all of them. The other case in which SSD efficiency might not occur is when the optimum in the minimisation of expected shortfall is zero, that is, $T_{1} \geq \lambda$. In this case the optimal solution may be ANY solution such that the corresponding funding ratio has all outcomes above the target $\lambda$. Adding a regularisation term ensures that the optimal solution is improved until SSD efficiency is achieved-an example of "better than target" situation. However, a regularisation term as in the SSD unscaled model involves the introduction of additional $S^{2}$ variables. Similarly to the previous subsection, we may add a term in the objective function such that, out of all solutions that minimise the expected shortfall below $\lambda$, the one with the highest expected value is chosen:

$$
\operatorname{Min} \frac{1}{S} \sum_{s=1}^{S} S h_{s}-\epsilon \sum_{s=1}^{S} F_{S}
$$

with $\epsilon$ a small enough number.

Secondly, the model that minimises expected shortfall below $\lambda$ is closely connected to an ICCP model (Klein Haneveld et al. 2010), in which the integrated chance constraint penalises shortfalls of the funding ratio distribution with respect to target $\lambda$. The connection is in the following sense. With the former, the expected shortfall is in the objective and a constraint on the terminal expected asset value is imposed. With the latter, the expected shortfall is the left hand side of a constraint, while maximising terminal expected asset value may be part of the objective. With appropriate choices of the right hand sides involved, the two models have the same optimal solution. We give an example of such a situation in the next section.

\section{Numerical experiment}

\subsection{Dataset, computational set up and motivation}

We consider a large defined benefit pension fund in Saudi Arabia, the General Organization for Social Insurance (GOSI) (http://www.gosi.gov.sa).

We consider a planning horizon of 10 years; $t=0$ refers to year 2016. We consider 16 asset classes: the Saudi equities represented by 15 sectors indices beside cash. Investment decisions have to be taken "now" $(t=0)$ and then rebalanced every year, $t=1 \ldots 9$. We generate a set of $S=300$ sample paths/scenarios for the asset returns, contributions and liability values at times $t=1 \ldots 10$. The scenarios for the asset returns are obtained by bootstrapping from historical data drawn from the Saudi Arabian stock market index (TASI) (https://www.tadawul.com.sa). For the riskfree rate of return (interest rate) we consider the current Saudi Arabian interest rate of $2 \%$ following (http://www.tradingeconomics.com/saudi-arabia/interest-rate), and 
assumed that it will stay at this level for the remaining of the investment period. The scenarios for the liability and contribution values have the same underlying source of uncertainty; they are generated based on populations models and a salary model, assuming that a fixed percentages of salaries are to be paid in, as contributions, or out, as liabilities. The dynamics of the pension's fund population is modelled by a BIDE (Birth, Immigration, Death, Emigration) model (Sandhya 2011). We used historical data on GOSI population as an input to this model (http://www.gosi.gov.sa); that includes the number of participants, employment and retirement rates for the last 10 years, salary average and average salary growth.

We implement the models (SSD-scaled) and (SSD-unscaled) developed in Sect. 3 with deterministic and non-deterministic target distributions of funding ratio. As a non-deterministic target distribution, we use a synthetic one with 300 equally likely scenarios, in each the lowest outcome is 0.9 and there is an increase by 0.0016 under each scenario. As a deterministic target distribution, we use one defined by the single outcome $\lambda=1.1$.

We refer to the SSD scaled model with deterministic target distribution as (Maximin); as exposed in Sect. 4.1, it is equivalent to a maximisation of the worst funding ratio.

We refer to the SSD unscaled model with deterministic target distribution as ICCP, because it has the same optimal solution as an ICCP of model in which the expected terminal asset value is maximised and constraint is imposed on the expected shortfall with respect to $\lambda$. We have showed in the previous section that, in case of deterministic target distribution with a single outcome, the SSD unscaled model reduces to a model in which the expected shortfall (below the single target) is minimised. If we constrain the expected shortafall to be below a specified limit (an ICCP type of model) we obtain the same optimal solutions, provided that the right hand sides are chosen appropriately. In our numerical experiments, we implement an ICCP model in which we maximise the expected terminal asset value and we constrain the expected shortfall to be no more than $5 \%$ of the lowest scenario value for liability at time 1 . We recorded this optimal value of the objective function; let us denote it by $A_{T}$. We implemented the SSD unscaled model with deterministic target 1.1 with a constraint on the expected terminal asset value: to be no less than $A_{T}$. The two models have the same optimal solution.

We have thus four SSD based models that we refer to as (SSD-scaled), (SSDunscaled), (Maximin) and (ICCP). In all four models, the right hand side of the constraint on the expected terminal asset value is the same (equal to $A_{T}$ which corresponds to a cumulated terminal wealth of 581.5548 billions of Saudi Riyals (SAR)). The value of $\epsilon$ is fixed to 0.0001. We implement the models in AMPL and solve them using CPLEX 12.5.1.0.

The objective of this computational work is to investigate differences and similarities in the quality of solutions obtained with the four models. More precisely, we look at the performance measures for the resulting return distributions and at the shape of the funding ratio distributions obtained in each of the four cases, particularly the left tails. We investigate whether by appropriately selecting a (non-deterministic) target distribution, improved funding ratio distributions can be obtained. Both in-sample and out-of-sample testing are performed. 
Table 1 Performance measures related to the returns for SSD-unscaled, SSD-scaled, ICCP, and maximin models

\begin{tabular}{lllll}
\hline Comparison criteria & SSD-unscaled & SSD-scaled & ICCP & Maximin \\
\hline Expected rate of return & $16.33 \%$ & $15.72 \%$ & $14.41 \%$ & $13.89 \%$ \\
$\begin{array}{l}\text { Sharpe ratio } \\
\text { Sortino ratio (computed with respect }\end{array}$ & 0.7757 & 0.7158 & 0.7656 & 0.7108 \\
$\quad$ to 0.02\% target rate of return) & 2.9161 & 3.8335 & 2.3188 & 3.8356 \\
VaR (5\%) & 0.103 & 0.085 & 0.1273 & 0.07 \\
CVaR (5\%) & 0.139 & 0.0922 & 0.1637 & 0.0754 \\
\hline
\end{tabular}

All the measures are calculated for the first time period

\subsection{Computational results}

Table 1 lists statistics and risk-adjusted performance measures of the rate of return of the portfolio such as: Sharpe ratio, Sortino ratio, Value at Risk (VaR) and Conditional Value at Risk (CVaR). For Sharpe and Sortino ratios, the target rate of return is set at $2 \%$, that is, excess rate of return and downside risk are calculated with respect to $2 \%$. $\mathrm{VaR}$ and $\mathrm{CVaR}$ are computed at parameter $5 \%$, that is, considering the worst $5 \%$ of the outcomes.

The results reinforce the similarity between (SSD-unscaled) and (ICCP), as well as between (SSD-scaled) and Maximin. The rate of returns of the (SSD-unscaled) and (ICCP) solutions have higher expected values and Sharpe ratios but have poorer statistics regarding left tails/unfavorable outcomes: VaR and CVaR are (much) higher, indicating larger losses under unfavorable scenarios, also the Sortino ratio is considerably lower, indicating poorer downside risk return adjusted performance. We notice that the (SSD-unscaled) solution performs better than the ICCP solution in all reported measures: expected value, risk-adjusted performance measures, left tail statistics.

The(SSD-scaled) and (Maximin) solutions are similar in that the statistics on left tails (as measured by $5 \% \mathrm{VaR}$ and $5 \% \mathrm{CVaR}$ ) and downside risk are considerably better at the expense of average performance. The (Maximin) solution has clearly the return distribution with the best left tail, but also with the lowest expected value. The (SSD-scaled) solution provides a compromise between acceptable left tails and higher expected value.

Table 2 lists the statistics of the funding ratio distributions, obtained with the four models considered. The $A \%$-scaled tail is the mean of the worst $A \%$ outcomes of the funding ratio.

The values illustrate well the main differences and similarities between the models - and also supports the motivation of this work. As before, the similarity between (SSD-unscaled) and (ICCP) models resides in a better overall performance at the expense of left tails/worst case scenarios. In contrast, (SSD-scaled) and (Maximin) solutions result in funding ratios with the best statistics for left tails (measured up to $25 \%$ of left tails). Particularly in the worst case scenarios, these models perform much better-the differences start to decrease as we move along the left tails and consider more outcomes of the distributions. As before, the solution of the (SSD scaled) 
Table 2 Performance measures related to the funding ratio for SSD-unscaled, SSD-scaled, ICCP, and maximin models

\begin{tabular}{lllll}
\hline Comparison criteria & SSD-unscaled & SSD-scaled & ICCP & Maximin \\
\hline Expected funding ratio & 1.160 & 1.153 & 1.141 & 1.135 \\
Minimum funding ratio & 0.8168 & 0.876 & 0.7926 & 0.8932 \\
$\begin{array}{l}\text { Expected shortfall of FR } \\
\text { with respect to 1.1 }\end{array}$ & 0.0455 & 0.0526 & 0.0430 & 0.0517 \\
$\begin{array}{l}\text { 1\%-Scaled tail } \\
\text { 5\%-Scaled tail }\end{array}$ & 0.8200 & 0.8794 & 0.7957 & 0.8967 \\
10\%-Scaled tail & 0.8526 & 0.8969 & 0.8283 & 0.9124 \\
15\%-Scaled tail & 0.8725 & 0.9165 & 0.8556 & 0.9290 \\
20\%-Scaled tail & 0.8946 & 0.9284 & 0.8915 & 0.9392 \\
25\%-Scaled tail & 0.9207 & 0.9383 & 0.9227 & 0.9471 \\
\hline
\end{tabular}

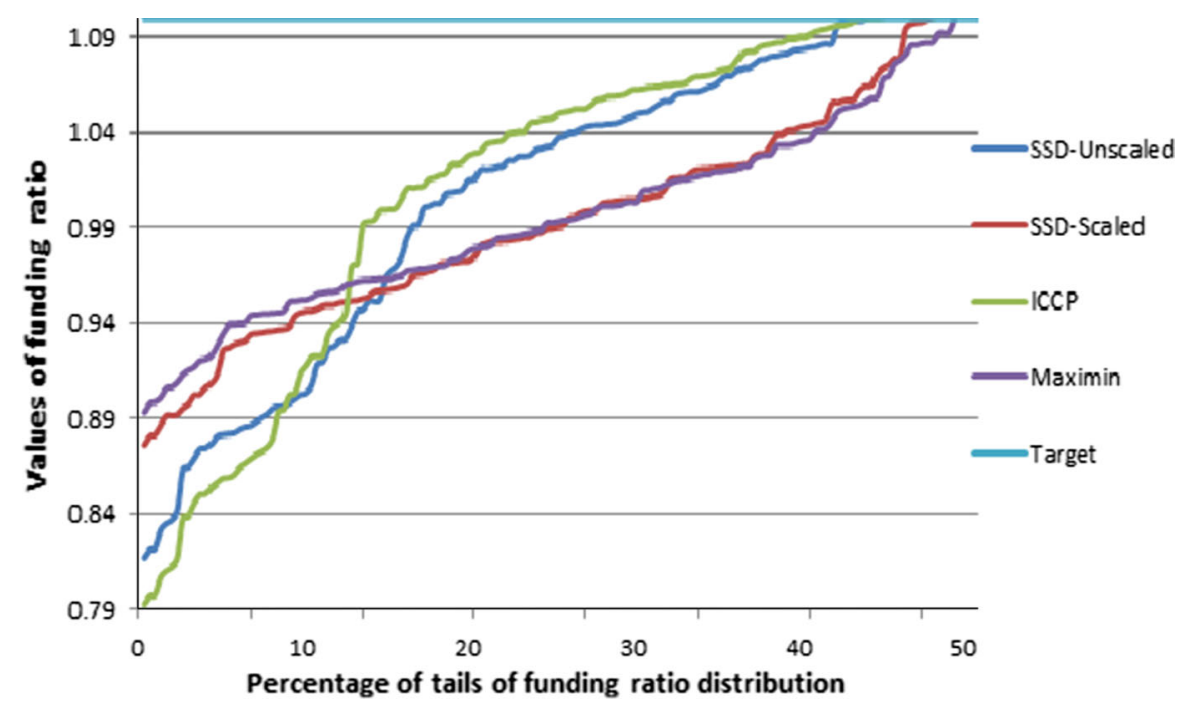

Fig. 1 Left tails of the funding ratio distributions corresponding to SSD-unscaled, SSD-scaled, ICCP and maximin models

model provides a compromise between reasonable left tail statistics and better overall/average performance. While the (SSD-unscaled) and (ICCP) solutions have rather similar characteristics, we note that the (SSD unscaled) solution results in better left tails, including higher minimum and even better average performance, at the expense of a marginal increase in expected shortfall below the target.

Figure 1 plots, for each of the four models, the left tails of the funding ratio distributions; more precisely, the outcomes of the funding ratio distributions that are below the target 1.1. The difference between the values illustrated in Table 2 and Fig. 1 is as follows. In Table 2, scaled tail values are reported while Fig. 1 displays actual values of the distributions. For example, the average of the worst $20 \%$ values of the ICCP 
Table 3 Out-of-sample analysis for the first stage decisions for the models: SSD-unscaled, SSD-scaled, ICCP, and maximin

\begin{tabular}{|c|c|c|c|c|}
\hline Comparison criteria & SSD-unscaled & SSD-scaled & ICCP & Maximin \\
\hline Expected rate of returns & $14.02 \%$ & $13.74 \%$ & $12.34 \%$ & $12.21 \%$ \\
\hline Sortino ratio & 1.5947 & 2.1166 & 1.3460 & 2.1986 \\
\hline Expected FR & 1.1198 & 1.1169 & 1.1036 & 1.1020 \\
\hline Minimum funding ratio & 0.5803 & 0.6516 & 0.5813 & 0.6903 \\
\hline $\begin{array}{l}\text { Expected shortfall of FR } \\
\text { with respect to } 1.1\end{array}$ & 0.0718 & 0.0720 & 0.0708 & 0.0699 \\
\hline $1 \%$-Scaled tail & 0.6527 & 0.7256 & 0.6450 & 0.7601 \\
\hline $5 \%$-Scaled tail & 0.7089 & 0.7810 & 0.6964 & 0.8103 \\
\hline $10 \%$-Scaled tail & 0.7534 & 0.8180 & 0.7396 & 0.8424 \\
\hline $15 \%$-Scaled tail & 0.7901 & 0.8448 & 0.7801 & 0.8650 \\
\hline $20 \%$-Scaled tail & 0.8243 & 0.8663 & 0.8211 & 0.8834 \\
\hline $25 \%$-Scaled tail & 0.8545 & 0.8847 & 0.8557 & 0.8991 \\
\hline
\end{tabular}

funding ratio distribution is 0.9227 . In Fig. 1, we can see that the worst $20 \%$ value of this distribution (the $20 \%$ quantile) is above 0.94 .

The main differences and similarities between the models are well illustrated in Fig. 1. The ICCP funding ratio "starts low" and it has the lowest outcomes up to $15 \%$ of the distribution. After this, it has the highest outcomes; hence overall it results in the lowest average shortfall below the target. The distributions corresponding to (SSD scaled) and (Maximin) are similar; the former starts lower but performs better in the rest of the distribution, although the differences are small. The (SSD-unscaled) distribution is closer in shape to the ICCP one; it has however higher outcomes under the worst $10 \%$ of scenarios.

We evaluate the first-stage decisions obtained by the above models out-of-sample over a large scenario set. Based on the recorded daily observations of Saudi stock market from Jun 2007 to Nov 2015, we obtain 1937 scenarios for the annual rates of returns of the component assets. Using the BIDE population model we generate 500 scenarios for the liabilities; we have thus 968,500 out-of-sample scenarios for the first time period. Table 3 illustrate the out-of-sample analysis of the first-stage decisions of the models (SSD-Unscaled, SSD-Scaled, ICCP and Maximin) over this data set.

It can be seen that the out-of sample results are mostly in line with the in-sample results, although (as expected) the worst case realisations are considerably lower, for all models considered. The solution of the Maximin model has the (out-of sample) funding ratio distribution with the highest worst case values and the highest left tails up to $25 \%$ of the distribution; on the other hand, the expected rate of return of the corresponding portfolio is the lowest, compared with the rest of the models. Interestingly, the solution of the ICCP model does not result in the distribution with the lowest expected shortfall-it is the Maximin model that does, although the difference is marginal. Similarly to the in-sample results, the (SSD-unscaled) and (SSD scaled) with non-deterministic target distributions have similar performances to ICCP and Maximin models, respectively, but do bring something new to the table. The solution 
of the (SSD unscaled) model improves on the very left tails of the funding ratio distribution, as compared to the ICCP model, while the solution of the (SSD scaled) model improves on the right tail/overall performance, compared to the Maximin model.

\section{Conclusions and further thoughts}

We have formulated ALM models in which the risk of underfunding is controlled using Second Order Stochastic Dominance. We obtain short-term funding ratio distributions that are SSD efficient, while a constraint is imposed on the expected terminal wealth. In addition to being SSD efficient, the funding ratio distribution comes close, in a well defined sense, to a benchmark distribution of funding ratio, whose outcomes are specified by the decision maker.

There are two main SSD models presented in this paper, a scaled model and an unscaled model. Progressively larger left tails of the funding ratio distribution are considered, either scaled (equivalent to averages of a progressively higher number of worst case values), or unscaled (equivalent to sums of a progressively higher number of worst case values). Target values are considered for scaled and unscaled tails; the worst difference between a tail and its corresponding target value is optimised. A regularisation term is added to ensure SSD efficiency in case of multiple optimal solutions.

Both models result in (possibly different) SSD efficient distributions of funding ratio. While the SSD unscaled model penalises outcomes of the funding ratio distribution below their targets in an evenly manner, with the SSD scaled model, the magnitude of shortfall below its target matters. A good way to grasp the difference between the models is by considering that in special cases, the SSD scaled model is equivalent to maximising the lowest funding ratio while the SSD unscaled model is equivalent to minimising the average of shortfalls below the target.

The advantage of using SSD models over previous approaches of imposing a risk constraint lies not only in better modelling of the (entire) funding ratio distribution. With a risk constraint on the funding ratio distribution, the decision maker has to set a right hand side, which in most cases is not straightforward; it can lead to infeasibility, or, in the opposite case, it may be under-restrictive. These issues are not encountered in the SSD models. Even if the target distribution has too high/unachievable values, the model is not infeasible. In the opposite case, if the target distribution has not "high" enough outcomes, the resulting distribution of funding ratio will be "better than target", that is, not just attain it, but improve on it until SSD efficiency is obtained.

A particular case with interesting connections to risk minimisation is obtained when the target distribution is deterministic, specified by a single outcome such as a required target funding ratio $\lambda$.

In most situations, the SSD scaled model is equivalent to a risk minimisation model, where risk is measured by the maximum loss. More precisely, the SSD scaled model can be reformulated as a (computationally much simpler) Maximin model which maximises the worst case value of the funding ratio.

The SSD unscaled model is equivalent in most cases to a risk minimisation model, where risk is measured by the lower partial moment of order 1 of the funding ratio 
around target $\lambda$, also called the expected shortfall below target $\lambda$. The well established ICCP model has the expected shortfall below target as a constraint, not in the objective function. By setting appropriate right hand side values, the SSD unscaled formulation and the ICCP formulation lead to the same optimal solutions.

There are situations in which the SSD models and risk minimisation models above may not be equivalent. This may happen when (a) the risk minimisation model has multiple optimal solutions; (b) in the case of minimisation of expected shortfall, the minimum is zero. In these cases, the optimal solution of the risk minimisation model is not guaranteed to be SSD efficient - unlike with the SSD formulations. A regularisation term should be added to the objective function in the risk minimisation models in order to guarantee SSD efficiency. However, this means increasing computational complexity to the level of the SSD formulations-for covering a very limited number of situations. In order to reduce the chance of the risk minimisation models resulting in an SSD dominated solution, we can add an extra term in the objective function representing the expected value of funding ratio, weighted by a very small number; this approach comes at no additional computational cost.

Thus, two established and computationally less expensive models, namely Maximin and ICCP, are particular cases of the SSD models developed in this paper. A natural question that arises is: can we obtain improved distributions of funding ratio by considering non-deterministic target distributions - and having thus the considerable extra computational difficulty of the generic SSD models? The computational study offers insight into this problem, by analysing solutions obtained from Maximin, ICCP and two SSD models (scaled and unscaled) with non-deterministic target distributions. The differences between the four solutions were rather small, however a few things can be pointed out. The ICCP solution, although with lowest expected shortfall below target, has the lowest left tails out of all solutions considered. The Maximin solution provides indeed the best outcome under the worst case scenario, however this advantage is not kept in the rest of the distribution. By using an SSD formulation, we may obtain better overall tails, at the expense of an only marginal decrease of worst case performance.

A possible strategy is to start by implementing either an ICCP or Maximin model and analyse the resulting distribution of funding ratio. Should this be not acceptable, one can implement a generic SSD model, by setting a (non-deterministic) target distribution based on the outcomes of the funding ratio already obtained. For example, the targets for the worst case scenario and the left tails can be increased, should these values be too low in the ICCP solutions. Similarly, the targets for the tails in the upper part of the distribution may be increased, should the Maximin model provide a solution with poor performance apart from worst case scenarios.

Open Access This article is distributed under the terms of the Creative Commons Attribution 4.0 International License (http://creativecommons.org/licenses/by/4.0/), which permits unrestricted use, distribution, and reproduction in any medium, provided you give appropriate credit to the original author(s) and the source, provide a link to the Creative Commons license, and indicate if changes were made. 


\section{References}

Bogentoft E, Romeijn HE, Uryasev S (2001) Asset/liability management for pension funds using CVaR constraints. J Risk Finance 3(1):57-71

Carino DR, Ziemba WT (1998) Formulation of the Russell-Yasuda Kasai financial planning model. Oper Res 46(4):433-449

Carino DR, Kent T, Myers DH, Stacy C, Sylvanus M, Turner AL, Watanabe K, Ziemba WT (1994) The Russell-Yasuda Kasai model: an asset/liability model for a Japanese insurance company using multistage stochastic programming. Interfaces 24(1):29-49

Charnes A, Cooper WW (1959) Chance-constrained programming. Manag Sci 6(1):73-79

Consigli G, Dempster MAH (1998) The CALM stochastic programming model for dynamic asset-liability management. Worldw Asset Liabil Model 10:464

de Oliveira AD, Filomena TP, Perlin MS, Lejeune M, de Macedo GR (2017) A multistage stochastic programming asset-liability management model: an application to the Brazilian pension fund industry. Optim Eng 18(2):349-368

Dempster MAH, Germano M, Medova EA, Villaverde M (2003) Global asset liability management. Br Actuar J 9(01):137-195

Dentcheva D, Ruszczynski A (2006) Portfolio optimization with stochastic dominance constraints. J Bank Finance 30(2):433-451

Dert C (1995) Asset liability management for pension funds: a multistage chance constrained programming approach. PhD thesis, Erasmus University, Rotterdam, The Netherlands

Dupačová J, Polívka J (2009) Asset-liability management for Czech pension funds using stochastic programming. Ann Oper Res 165(1):5-28

Escudero LF, Garín MA, Merino M, Pérez G (2016) On time stochastic dominance induced by mixed integer-linear recourse in multistage stochastic programs. Eur J Oper Res 249(1):164-176

Fábián CI, Mitra G, Roman D (2011a) Processing second-order stochastic dominance models using cuttingplane representations. Math Program 130(1):33-57

Fábián CI, Mitra G, Roman D, Zverovich V (2011b) An enhanced model for portfolio choice with SSD criteria: a constructive approach. Quant Finance 11(10):1525-1534

Fishburn PC (1977) Mean-risk analysis with risk associated with below-target returns. Am Econ Rev 67(2):116-126

Gallo A (2009) Risk management and supervision for pension funds: critical implementation of ALM models. PhD thesis, Università degli Studi di Napoli Federico II

Geyer A, Ziemba WT (2008) The innovest Austrian pension fund financial planning model InnoALM. Oper Res 56(4):797-810

Kallberg JG, White RW, Ziemba WT (1982) Short term financial planning under uncertainty. Manag Sci 28(6):670-682

Klein Haneveld WK (1986) Duality in stochastic linear and dynamic programming. Lecture notes in economics and mathematical systems, vol 274. Springer, Berlin

Klein Haneveld WK, Van Der Vlerk MH (2006) Integrated chance constraints: reduced forms and an algorithm. CMS 3(4):245-269

Klein Haneveld WK, Streutker MH, Van Der Vlerk MH (2010) An ALM model for pension funds using integrated chance constraints. Ann Oper Res 177(1):47-62

Kopa M, Chovanec P (2008) A second-order stochastic dominance portfolio efficiency measure. Kybernetika 44(2):243-258

Kopa M, Post T (2015) A general test for SSD portfolio efficiency. OR Spectr 37(3):703-734

Kopa M, Moriggia V, Vitali S (2018) Individual optimal pension allocation under stochastic dominance constraints. Ann Oper Res 260(1-2):255-291

Kouwenberg R (2001) Scenario generation and stochastic programming models for asset liability management. Eur J Oper Res 134(2):279-292

Kusy MI, Ziemba WT (1986) A bank asset and liability management model. Oper Res 34(3):356-376

Mulvey JM, Gould G, Morgan C (2000) An asset and liability management system for Towers PerrinTillinghast. Interfaces 30(1):96-114

Ogryczak W, Ruszczynski A (2002) Dual stochastic dominance and related mean-risk models. SIAM J Optim 13(1):60-78 
Pirbhai M, Mitra G, Kyriakis T (2003) Asset liability management using stochastic programming. Technical report, The Centre for the Analysis of Risk and Optimisation Modelling Applications (CARISMA), Brunel University, London. http://bura.brunel.ac.uk/handle/2438/748

Post T, Kopa M (2013) General linear formulations of stochastic dominance criteria. Eur J Oper Res 230(2):321-332

Rockafellar RT, Uryasev S (2000) Optimization of conditional value-at-risk. J Risk 2:21-42

Roman D, Darby-Dowman K, Mitra G (2006) Portfolio construction based on stochastic dominance and target return distributions. Math Program 108(2-3):541-569

Roy AD (1952) Safety first and the holding of assets. Econometrica 20(3):431-449

Sandhya A (2011) Models for population growth. Biotech Articles. http://www.biotecharticles.com/OthersArticle/Models-For-Population-Growth-758.html

Saudi Stock Exchange. https://www.tadawul.com.sa

The General Organization for Social Insurance (GOSI). http://www.gosi.gov.sa

Trading Economics. http://www.tradingeconomics.com/saudi-arabia/interest-rate

Whitmore GA, Findlay MC (1978) Stochastic dominance: an approach to decision-making under risk. Lexington Books, Lanham

Wierzbicki A (1982) A mathematical basis for satisficing decision making. Math Model 3(5):391-405

Yang X, Gondzio J, Grothey A (2010) Asset liability management modelling with risk control by stochastic dominance. J Asset Manag 11(2):73-93

Young MR (1998) A minimax portfolio selection rule with linear programming solution. Manag Sci 44(5):673-683 